\title{
Vibration Properties of a Steel-PMMA Composite Beam
}

\author{
Yuyang $\mathrm{He}^{1,2}$ and Xiaoxiong Jin ${ }^{1}$ \\ ${ }^{1}$ Automotive College, Tongji University, Shanghai 201804, China \\ ${ }^{2}$ Jiangsu College of Information Technology, Wuxi 214153, China
}

Correspondence should be addressed to Yuyang He; wxheyy@126.com

Received 25 October 2014; Revised 1 February 2015; Accepted 2 March 2015

Academic Editor: Ahmet S. Yigit

Copyright (C) 2015 Y. He and X. Jin. This is an open access article distributed under the Creative Commons Attribution License, which permits unrestricted use, distribution, and reproduction in any medium, provided the original work is properly cited.

\begin{abstract}
A steel-polymethyl methacrylate (steel-PMMA) beam was fabricated to investigate the vibration properties of a one-dimensional phononic crystal structure. The experimental system included an excitation system, a signal acquisition system, and a data analysis and processing system. When an excitation signal was exerted on one end of the beam, the signals of six response points were collected with acceleration sensors. Subsequent signal analysis showed that the beam was attenuated in certain frequency ranges. The lumped mass method was then used to calculate the bandgap of the phononic crystal beam to analyze the vibration properties of a beam made of two different materials. The finite element method was also employed to simulate the vibration of the phononic crystal beam, and the simulation results were consistent with theoretical calculations. The existence of the bandgap was confirmed experimentally and theoretically, which allows for the potential applications of phononic crystals, including wave guiding and filtering, in integrated structures.
\end{abstract}

\section{Introduction}

Phononic crystals are synthetic materials formed by a periodical variation of acoustic properties. The existence of bandgap in phononic crystals has been confirmed through experiments [1-3]. It is also known that bandgap can prevent phonons with certain ranges of frequencies from being transmitted through the material. This property allows it to be used for isolation and noise reduction [4-7]. Additionally, other potential applications, including sensors and filters, have been found [8].

Based on the relationship between its bandgap wavelength and lattice constant, a phononic crystal can be divided into two types: the Bragg scattering type and the locally resonant type. For the Bragg scattering type, its bandgap wavelength and lattice constant are of the same order of magnitude $[9,10]$.

The property of the vibration bandgap is related to the elastic constants, density, and sound velocity of complex media components, filling fractions of the components as well as the lattice structure and size. The creation of the vibration bandgap occurs due to the difference between the elastic constants of the elements.
As for locally resonant phononic crystals, their properties are determined by the strong resonance of a locally resonant unit. The resonance of a single scattering body is excited by elastic waves of a certain frequency, which then interacts with the incident wave and stops the spreading. The bandgap is dependent on the structure of the scatterer and its interaction with various elastic waves [11, 12].

Due to the existence of bandgaps, phonons with certain ranges of frequencies can be prevented from transmitting. By appropriately choosing the materials of the phononic crystal, including the scatterer, matrix, and coating, a desired bandgap can be obtained, which implies its potential application in low-frequency noise and vibration control for machinery and equipment.

\section{Experimental}

2.1. Preparation of Specimen. In this work, steel and polymethyl methacrylate (PMMA) were chosen to be used to make the phononic crystal beam because of the large differences between their elastic constants. The steel was machined by a lathe into cylinders with diameters of $30 \mathrm{~mm}$ 
TABLE 1: Comparison of the attenuation of the output points.

\begin{tabular}{lcc}
\hline Point position & Root mean square $(\mathrm{dB})$ & Average $(\mathrm{dB})$ \\
\hline Point 1 & 20.61 & 24.25 \\
Point 2 & 20.73 & 24.66 \\
Point 3 & 21.06 & 25.32 \\
Point 4 & 22.55 & 26.85 \\
Point 5 & 22.39 & 26.26 \\
Point 6 & 14.61 & 19.14 \\
\hline
\end{tabular}

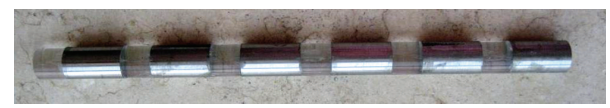

FIGURE 1: The experimental phononic crystal beam made of PMMA and steel.

and lengths of $50 \mathrm{~mm}$. The PMMA was made into cylinders with diameters of $30 \mathrm{~mm}$ and lengths of $25 \mathrm{~mm}$.

The specimen of the phononic crystal included six segments of alternating steel and PMMA cylinders with a total length of $450 \mathrm{~mm}$, as shown in Figure 1. Additionally, silicone was used to glue the cylinders together, followed by room temperature cooling for 12 hours.

2.2. Test System and Method. The excitation and signal acquisition system included a set of exciters, acceleration sensors, power amplifiers analyzers, and the test software. LMS software was employed to test and analyze the vibration acceleration signals as a function of the position. And the frequency response function was eventually obtained at a certain position in the specimen.

A broadband white noise signal is firstly generated from the signal generator module system and is then amplified by the power amplifier before driving the vibration exciter to generate a corresponding signal. As a result, the vertical vibration bending elastic wave can be excited on the phononic crystal beam. Six acceleration sensors were installed on different vibration signal acquisition positions, as shown in Figure 2. The acceleration sensors were numbered 1 to 6 and used to detect the acceleration signal when the vibration signal was applied to one end of the phononic crystal beam.

In the experimental setup, as shown in Figure 3, the specimen was hung with two soft rubber ropes in order to guarantee its free boundary conditions. A noise excitation signal with a frequency range of 0 to $25 \mathrm{kHz}$ was excited on the bottom of one end of the beam, while the acceleration sensors were used to collect the signal for analysis and comparison.

2.3. Test Results and Discussion. The results of the transmission test are shown in Figure 4 with the transmission characteristic curves of Points 1 to 6, as marked in Figure 2.

As can be seen in Figure 4, different attenuation levels of the acceleration signal were observed for different points in the entire frequency range. A comparison of the attenuation levels of each point is shown in Table 1. By comparing the average attenuation values, it could be concluded that the attenuation gradually increased from Point 1 to Point 4 ,

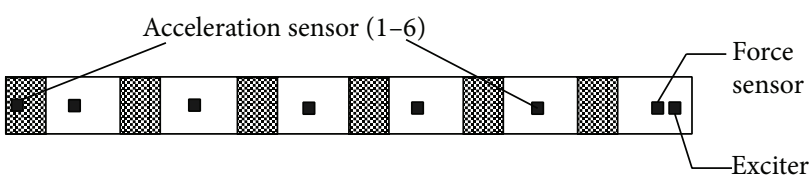

FIGURE 2: Sensor arrangement of the phononic crystal beam.

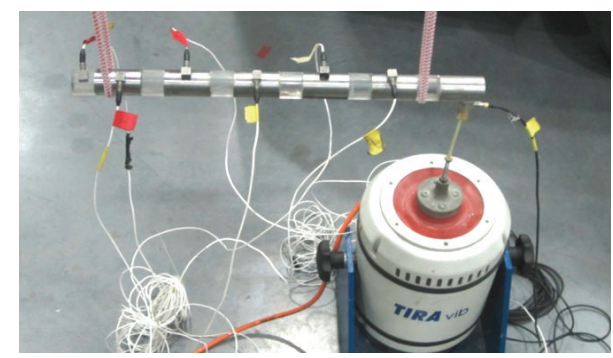

FIGURE 3: Experimental setup for the transmission characteristic test.

with Point 4 achieving a maximum attenuation of $26.85 \mathrm{~dB}$. For Point 4, the damping characteristics can be seen in the frequency range from 7.1 to $14.2 \mathrm{kHz}$ and from 15 to $24.7 \mathrm{kHz}$.

\section{Theoretical Analyses}

3.1. Calculation Using the Lumped Mass Method. Theoretically, the vibration property of the phononic crystal beam can be calculated. In this work, the lumped mass method was employed to calculate the bandgap by discretizing the continuous medium into elements. As a result, the band structure of the continuous media crystal was obtained by calculating the band structure of the discrete system, which contained several concentrated mass and massless springs as the connections between them [13].

One-dimensional phononic crystal beams composed of two different materials alternately arranged in the $x$ direction were formed, a structural model of which is shown in Figure 5(a), where $a$ represents a unit of phononic crystal beam. Taking one unit as an example, Figure 5(b) shows the discretion of one unit. According to the idea of the lumped mass method, the continuous medium was divided into a finite number of lumped masses, and the connections between the simplified lumped mass and mass-less spring connection, a unit composed of two materials, were reduced to a finite number of degrees of freedom of spring oscillator structures. One-dimensional phononic crystals can be simplified to an infinite periodic mass-spring structure.

This unit was simplified into a spring oscillator, consisting of a concentrated mass and a spring component $[14,15]$. The length of each spring oscillator was denoted as $d$, which was calculated from the ratio of the lattice constant $a$ and the number of spring oscillators $n$.

As mentioned in Section 2.1, the lengths of the steel and PMMA cylinders were 0.05 and $0.025 \mathrm{~m}$; thus the lattice constant of each unit of the cylinder was $0.075 \mathrm{~m}$. Detailed performance parameters of the materials are listed in 


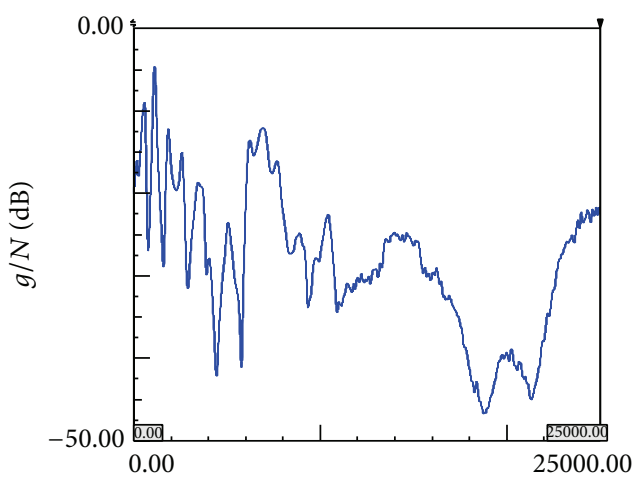

$(\mathrm{Hz})$

$\square \quad F$

FRF Point 6/Point 5

\begin{tabular}{|c|c|c|c|c|c|}
\hline Curve & 0.00 & 25000.00 & Average & RMS & $\mathrm{Hz}$ \\
\hline
\end{tabular} \begin{tabular}{|l|l|l|l|l|l|}
\hline- & -20.06 & -21.71 & -24.25 & -20.61 & $\mathrm{~dB}$ \\
\hline
\end{tabular}

(a) Point 1

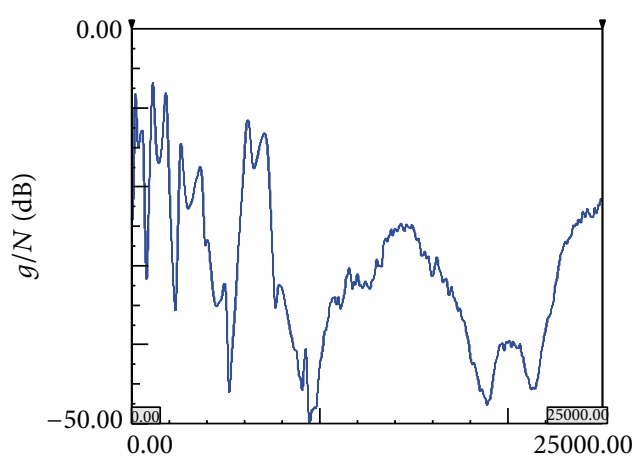

$(\mathrm{Hz})$

$F$

— FRF Point 8/Point 5

\begin{tabular}{|c|c|c|c|c|c|}
\hline Curve & 0.00 & 25000.00 & Average & RMS & Hz \\
\hline- & -23.92 & -21.70 & -25.32 & -21.06 & dB \\
\hline
\end{tabular}

(c) Point 3

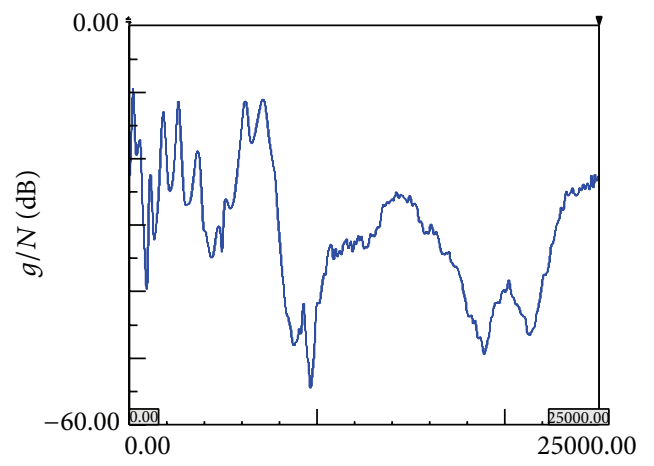

$(\mathrm{Hz})$

$F$

- FRF Point 10/Point 5

\begin{tabular}{|c|c|c|c|c|c|}
\hline Curve & 0.00 & 25000.00 & Average & RMS & $\mathrm{Hz}$ \\
\hline- & -22.55 & -22.59 & -26.26 & -22.39 & $\mathrm{~dB}$ \\
\hline
\end{tabular}

(e) Point 5

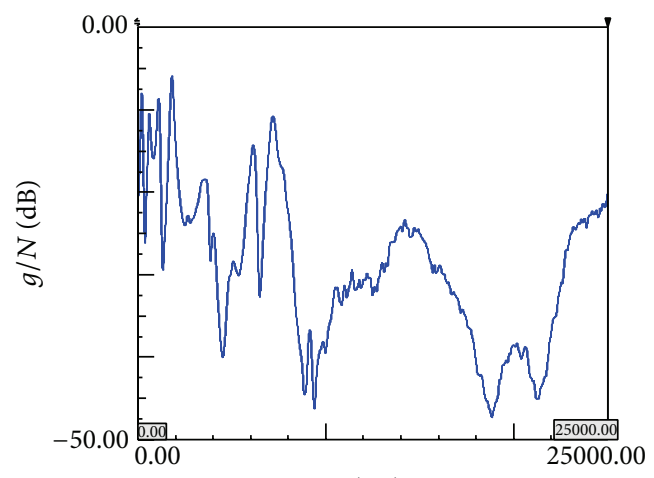

$(\mathrm{Hz})$

口 $F$

— FRF Point 7/Point 5

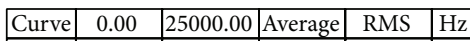

\begin{tabular}{l|l|l|l|l|l|}
\hline- & -21.09 & -20.21 & -24.66 & -20.73 & $\mathrm{~dB}$ \\
\hline
\end{tabular}

(b) Point 2

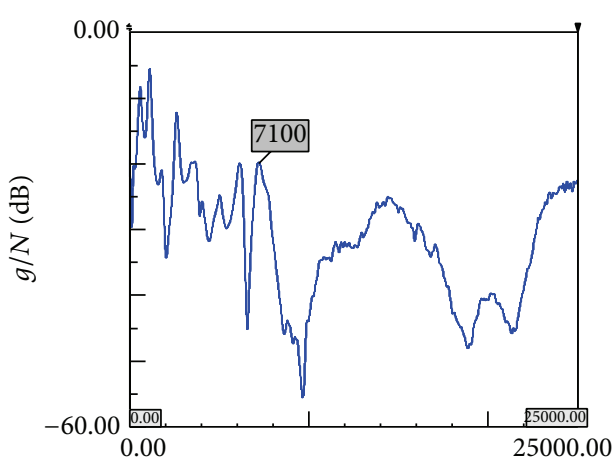

$(\mathrm{Hz})$

ㅁ $F$

- FRF Point 9/Point 5

\begin{tabular}{|c|c|c|c|c|c|}
\hline Curve & 0.00 & 25000.00 & Average & RMS & $\mathrm{Hz}$ \\
\hline- & -19.37 & -22.44 & -26.85 & -22.55 & $\mathrm{~dB}$ \\
\hline
\end{tabular}

(d) Point 4

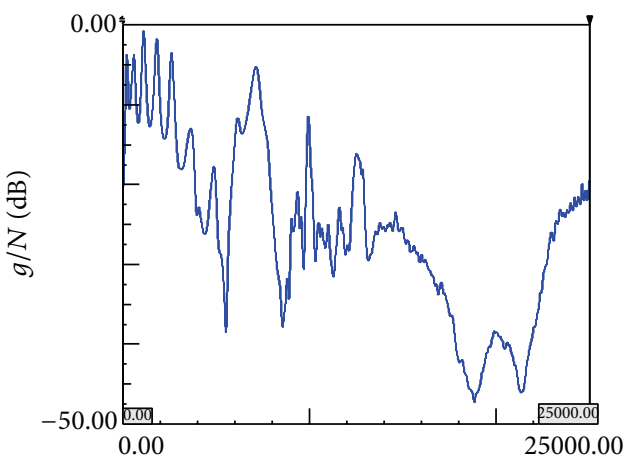

$(\mathrm{Hz})$

- $F$

- FRF Point 11/Point 5

\begin{tabular}{|c|c|c|c|c|c|}
\hline Curve & 0.00 & 25000.00 & Average & RMS & $\mathrm{Hz}$ \\
\hline- & -21.50 & -19.55 & -19.14 & -14.61 & $\mathrm{~dB}$ \\
\hline
\end{tabular}

(f) Point 6

FIGURE 4: Transmission characteristic curve of phononic crystals at the output points. 
TABLE 2: Performance parameters of materials.

\begin{tabular}{lccccc}
\hline Material name & $\begin{array}{c}\text { Density }(\rho) \\
\mathrm{Kg} / \mathrm{m}^{3}\end{array}$ & $\begin{array}{c}\text { Modulus }(E) \\
(\mathrm{pa})\end{array}$ & Poisson's ratio & \multicolumn{2}{c}{$\begin{array}{c}\text { Lame coefficients } \\
\lambda(\mathrm{pa})\end{array}$} \\
\hline Steel & 7840 & $2.16 \times 10^{11}$ & 0.28 & $1.002 \times 10^{10}$ & \multicolumn{2}{c}{$\begin{array}{c}\text { Lame coefficients } \\
(\mathrm{pa})\end{array}$} \\
PMMA & 1300 & $1.37 \times 10^{5}$ & 0.47 & $3.87 \times 10^{9}$ & $8.29 \times 10^{11}$ \\
Aluminum & 2799 & $7.21 \times 10^{10}$ & 0.344 & $5.43 \times 10^{10}$ & $1.2 \times 10^{9}$ \\
Epoxy & 1142 & $2.9 \times 10^{9}$ & 0.378 & $3.2602 \times 10^{9}$ & $2.56 \times 10^{10}$ \\
\hline
\end{tabular}

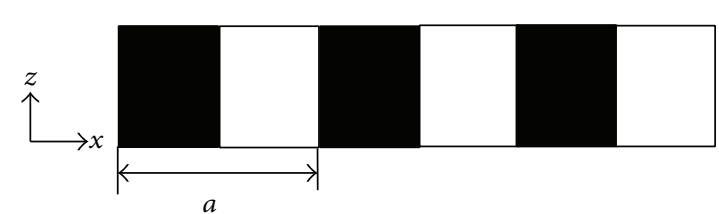

(a) Structural model of a one-dimensional phononic crystal

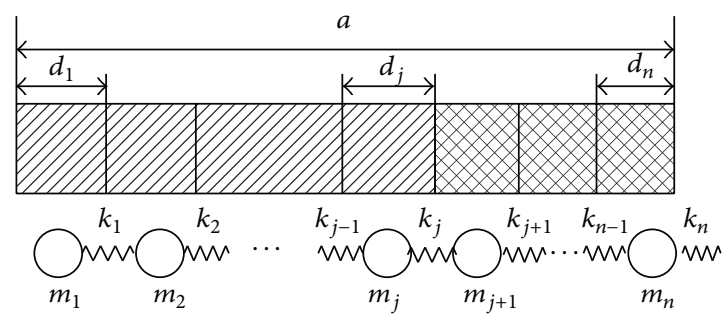

(b) The discrete case of a unit

FIGURE 5: One-dimensional phononic crystal unit.

Table 2. For a comparison of band structure, the parameters of aluminum and epoxy are also listed.

Assuming the two continuous media are ideal springs that only contain one material with a length of $d_{j}$ for each discrete element, the structure parameter $m$ of a spring oscillator is given by

$$
m_{j}=\rho S d_{j}, \quad j=1, \ldots, n,
$$

where $S$ is the cross-sectional area of the phononic crystal and $\rho$ is the density of the discrete element.

For each half of the discrete element, the normal stresses along the $x$ direction and shear stress along the $z$ direction are proportional to the strain, which gives

$$
\begin{gathered}
\frac{F_{i}^{x}}{S}=(\lambda+2 \mu) \frac{\Delta x}{d_{j} / 2}, \\
\frac{F_{i}^{z}}{S}=\mu \frac{\Delta z}{d_{j} / 2},
\end{gathered}
$$

where $\Delta x$ and $\Delta z$ are the tensile and shear displacements along the $x$ and $z$ direction, respectively, and $\lambda, \mu$ are the Lame constants of the material.

The spring between the adjacent oscillators can be regarded as two springs connected in a series. If the materials between the two adjacent discrete elements are different, the tensile stiffness along the $x$ direction and the shear stiffness along the $z$ direction can be described as

$$
\begin{gathered}
k_{i}^{x}=\frac{2\left(\lambda_{A}+2 \mu_{A}\right)\left(\lambda_{B}+2 \mu_{B}\right) S}{\left(\lambda_{A}+2 \mu_{A}\right) d_{j+1}+\left(\lambda_{B}+2 \mu_{B}\right) d_{j}}, \\
k_{i}^{z}=\frac{2 \mu_{A} \mu_{B} S}{\mu_{A} d_{j+1}+\mu_{B} d_{j}} .
\end{gathered}
$$

The unit of a one-dimensional Bragg scatting phononic crystal includes $n$ springs from $k_{1}$ to $k_{n}$ and $n$ spring

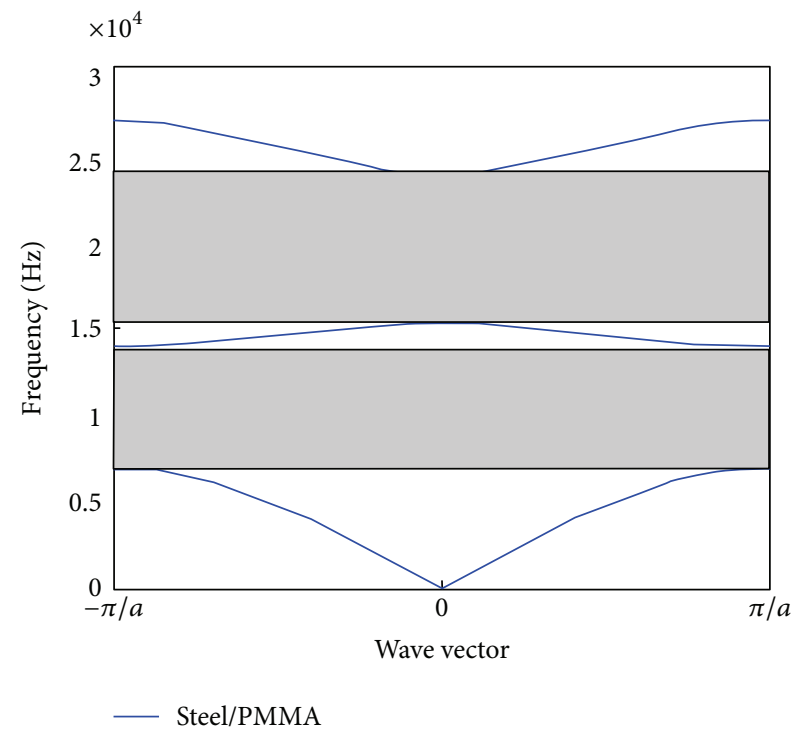

FIGURE 6: Calculation of band structure with lumped mass method.

oscillators connected in a series with a mass from $m_{1}$ to $m_{n}$. In this situation, $d_{j}$ is used to denote the distance between the spring oscillators. The lattice constant remains unchanged while $x_{j}$ is the displacement of the corresponding lumped mass.

The longitudinal motion equation of point $j$ is given by

$$
m_{j} \ddot{z}_{j}=k_{j}\left(z_{j+1}-z_{j}\right)-k_{j-1}\left(z_{j}-z_{j-1}\right), \quad j=1, \ldots, n .
$$

According to the Bloch theorem, with a periodic boundary, the particle motion equation is as follows:

$$
z_{j}=A_{j} e^{i\left(q \sum_{j=1}^{j} d_{j}-\omega t\right)},
$$




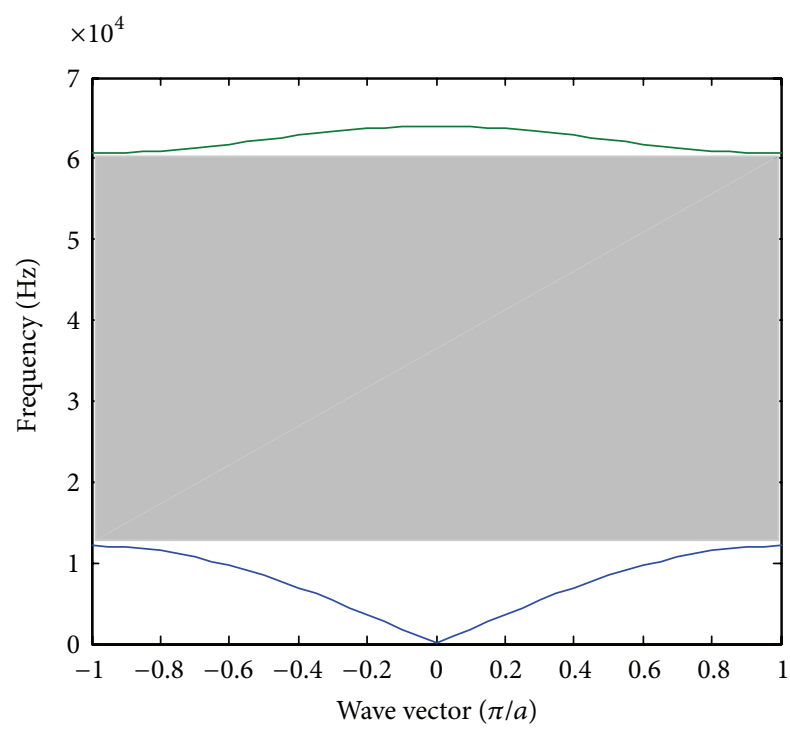

FIgURE 7: Band structure (steel-epoxy beam).

where $A_{j}$ is the amplitude, $\omega$ is the angular frequency, $q \sum_{j=1}^{j} d_{j}$ is a phase factor of oscillator $j$, and $q$ is the wave vector, whose value is in the first Brillouin zone $(-\pi / a$ to $\pi / a$, where $a$ is the lattice constant $t$ ). By substituting (5), (4) can be simplified into

$$
\left(\frac{k_{j}+k_{j-1}}{m_{j}}-\omega^{2}\right) A_{j}=\frac{k_{j}}{m_{j}} e^{i q d_{j+1}} A_{j+1}+\frac{k_{j-1}}{m_{j}} e^{-i q d_{j}} A_{j-1} .
$$

Due to the spring oscillators being arranged in a periodic way, the equation of the periodic boundary is as follows:

$$
\begin{aligned}
k_{0}=k_{n}, & k_{1}=k_{n+1}, \\
m_{0}=m_{n}, & m_{1}=m_{n+1}, \\
d_{0}=d_{n}, & d_{1}=d_{n+1}, \\
A_{0}=A_{n}, & A_{1}=A_{n+1} .
\end{aligned}
$$

By substituting (7), (6) is expressed in the matrix form as

$$
\left(X(q)-\omega^{2} I\right) A=0,
$$

where

$$
X(q)=\left(\begin{array}{cccccc}
\frac{k_{1}+k_{n}}{m_{1}} & -\frac{k_{1}}{m_{1}} e^{i q d_{2}} & 0 & \cdots & 0 & -\frac{k_{n-1}}{m_{1}} e^{-i q d_{n}} \\
-\frac{k_{1}}{m_{2}} e^{-i q d_{2}} & \frac{k_{1}+k_{2}}{m_{2}} & -\frac{k_{2}}{m_{2}} e^{i q d_{3}} & 0 & \ldots & 0 \\
\vdots & \vdots & \vdots & \vdots & \vdots & \vdots \\
-\frac{k_{n}}{m_{n}} e^{i q d_{1}} & 0 & \cdots & 0 & -\frac{k_{n-1}}{m_{n}} e^{-i q d_{n}} & \frac{k_{n}+k_{n-1}}{m_{n}}
\end{array}\right) .
$$

The spring stiffness is given by

$$
\begin{gathered}
k_{1}^{z}=k_{2}^{z}=\frac{\mu_{s} S_{s}}{d}, \quad k_{4}^{z}=\frac{\mu_{e} S_{e}}{d}, \\
k_{3}^{z}=k_{5}^{z}=\frac{2 \mu_{s} 2 \mu_{e} S}{\left(\mu_{s}+\mu_{e}\right) d} .
\end{gathered}
$$

Calculated with the lumped mass method above, the band structure of the phononic crystal of steel and PMMA was as shown in Figure 6. The bandgap was in the range of 7.0$14.7 \mathrm{kHz}$ and $15.2-24.8 \mathrm{kHz}$.

In order to investigate the band structures of different materials, the steel and PMMA were replaced with aluminum and epoxy. The parameters of the materials are shown in Table 2. The two band structures are shown in Figures 7 and 8.

Figure 7 shows the band structure of a one-dimensional beam made of steel and epoxy, whose frequency range of bandgap was from 11.679 to $58.526 \mathrm{kHz}$. Figure 8 shows the band structure of a one-dimensional beam made of aluminum and epoxy whose frequency range of bandgap was from 2.5 to $6.2 \mathrm{kHz}$. Therefore, it could be concluded that different frequency ranges of bandgap can be obtained when the materials used to make the beam are different.

3.2. Finite Element Calculation. Commercial finite element software was used to calculate the transmission characteristics to obtain reliable results. The finite element method was also based on the lumped mass algorithm, and it allowed easy calculations for complicated structures.

The geometry of the phononic crystal beam was established using Patran software with a total number of 5760 elements meshed as tria3, as shown in Figure 9. The beam was assumed to be in a free standing state. The excitation was exerted on one end of the phononic crystal beam in the vertical direction, and the response signal was collected on the other end of the beam. The transmission characteristics of the crystal structure obtained from the MSC Nastran software are shown in Figure 10.

In Figure 10, there are two large transmission attenuation regions of frequency at $6.8 \sim 14.2 \mathrm{kHz}$ and $15.8 \sim 25.2 \mathrm{kHz}$, which correspond to the bandgaps shown in Figure 6. 


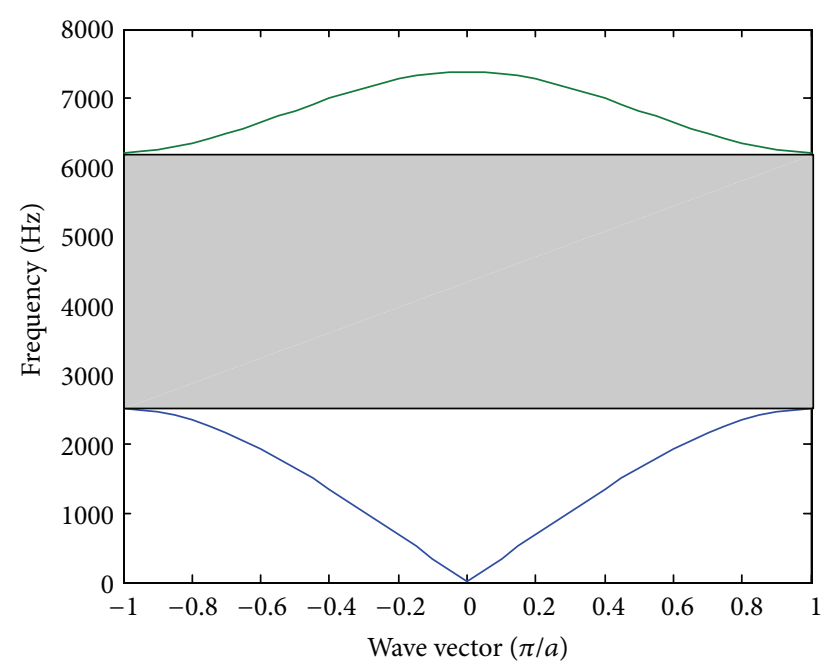

FIGURE 8: Band structure (aluminum-epoxy beam).

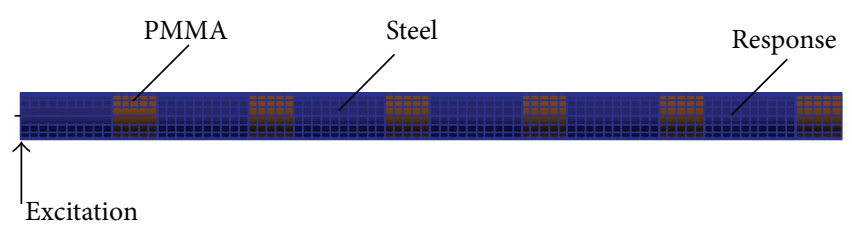

FIGURE 9: Finite element model of the phononic crystals beam.

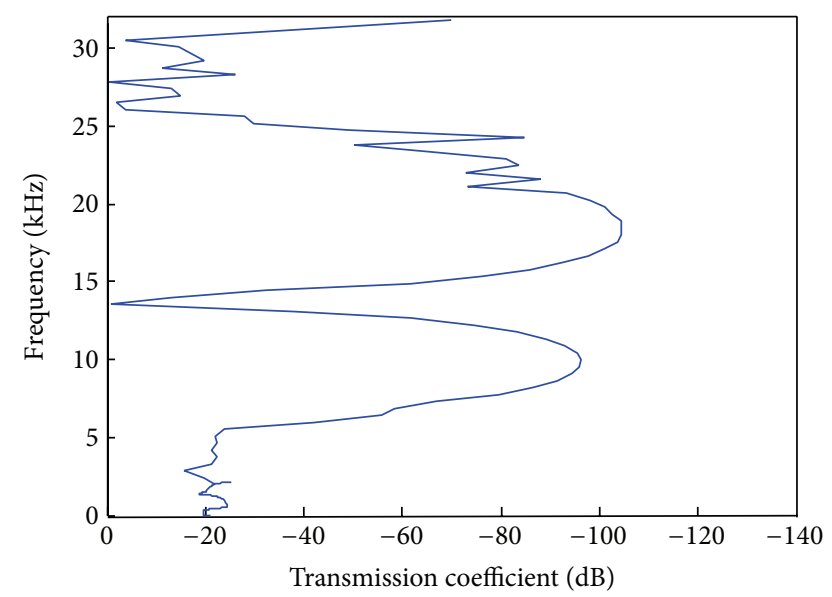

FIGURE 10: Transmission characteristics of phononic crystal beam.

The results of the finite element calculation of frequency range were the same as the lumped mass methods.

\section{Conclusions}

A specimen of a phononic crystal beam was fabricated using steel and PMMA, and the propagation curves of the acceleration response were obtained. As revealed by the experiments, the average attenuation was $26.85 \mathrm{~dB}$. The lumped mass method was also used to calculate the band structures of beams consisting of different materials such as steel-PMMA, steel-epoxy, and aluminum-epoxy. It was discovered that different frequencies of bandgaps could be obtained from beams made of different materials. The bandgap of the phononic crystal beam made of steel and PMMA was calculated using the finite element method, which was consistent with the results from the lumped mass method.

\section{Conflict of Interests}

The authors declare that there is no conflict of interests regarding the publication of this paper.

\section{References}

[1] G. Acar and C. Yilmaz, "Experimental and numerical evidence for the existence of wide and deep phononic gaps induced by inertial amplification in two-dimensional solid structures," Journal of Sound and Vibration, vol. 332, no. 24, pp. 6389-6404, 2013.

[2] M. Bavencoffe, B. Morvan, A.-C. Hladky-Hennion, and J.L. Izbicki, "Experimental and numerical study of evanescent waves in the mini stopband of a $1 \mathrm{D}$ phononic crystal," Ultrasonics, vol. 53, no. 2, pp. 313-319, 2013.

[3] J. Ryu, Y. K. Ju, S. W. Yoon, and S. D. Kim, "Bending capacity of glass fibre steel composite plate (GSP) reinforced composite slab," Materials Research Innovations, vol. 17, supplement 2, pp. S27-S34, 2013.

[4] M. M. Sigalas and E. N. Economou, "Elastic and acoustic wave band structure," Journal of Sound and Vibration, vol. 158, no. 2, pp. 377-382, 1992.

[5] M. S. Kushwaha, P. Halevi, L. Dobrzynski, and B. DjafariRouhani, "Acoustic band structure of periodic elastic composites," Physical Review Letters, vol. 71, no. 13, pp. 2022-2025, 1993.

[6] J. O. Vasseur, B. Djafari-Rouhani, L. Dobrzynski, M. S. Kushwaha, and P. Halevi, "Complete acoustic band gaps in periodic fibre reinforced composite materials: the carbon/epoxy composite and some metallic systems," Journal of Physics: Condensed Matter, vol. 6, no. 42, pp. 8759-8770, 1994.

[7] R. Martínez-Sala, J. Sancho, J. V. Sánchez, V. Gómez, J. Llinares, and F. Meseguer, "Sound attenuation by sculpture," Nature, vol. 378, no. 6554, p. 241, 1995.

[8] A. Oseev, M. Zubtsov, and R. Lucklum, "Gasoline properties determination with phononic crystal cavity sensor," Sensors and Actuators, B: Chemical, vol. 189, pp. 208-212, 2013.

[9] X. S. Wen, J. H. Wen, D. L. Yu et al., Phononic Crystal, National Defense Industry Press, 2009.

[10] M. Kafesaki, M. M. Sigalas, and N. García, "Frequency modulation in the transmittivity of wave guides in elastic-wave bandgap materials," Physical Review Letters, vol. 85, no. 19, pp. 40444047, 2000.

[11] S.-W. Zhang and J.-H. Wu, "Low-frequency band gaps in phononic crystals with composite locally resonant structures," Acta Physica Sinica, vol. 62, no. 13, Article ID 134302, 2013.

[12] Z. Liu, C. T. Chan, P. Sheng, A. L. Goertzen, and J. H. Page, "Elastic wave scattering by periodic structures of spherical objects: theory and experiment," Physical Review B-Condensed Matter and Materials Physics, vol. 62, no. 4, pp. 2446-2457, 2000.

[13] J.-G. Hu, J. Zhang, X. Zhang, and J.-M. Guo, "Band gaps and application of one-dimensional phononic crystals consisted of granite and nitrile rubber," Journal of Yunnan University (Natural Sciences), vol. 28, no. 6, pp. 504-508, 2006. 
[14] J.-H. Wen, G. Wang, Y.-Z. Liu, and H.-G. Zhao, "Research on vibration band gaps of one dimensional phononic crystals consisted of metal and nitrile butadiene rubber," Journal of Vibration Engineering, vol. 18, no. 1, pp. 1-7, 2005.

[15] J. H. Wen, G. Wang, Y. Z. Liu et al., "Lumped-mass method on calculation of elastic band gaps of one-dimensional phononic crystals," Acta Physica Sinica, vol. 53, pp. 3384-3333, 2004. 

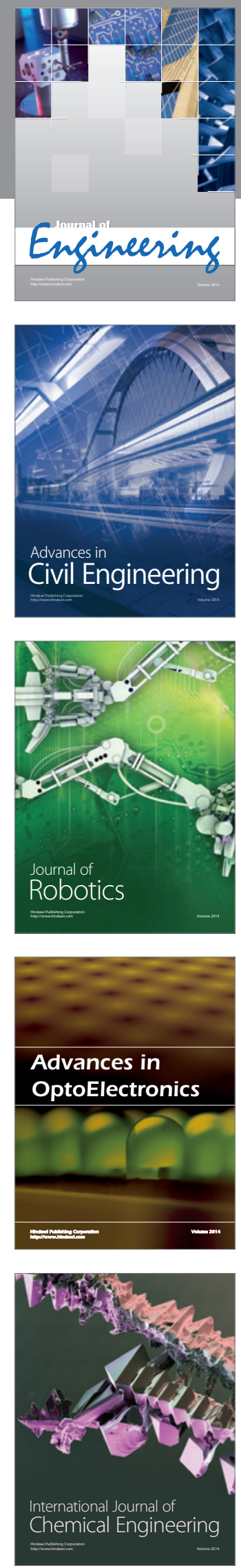

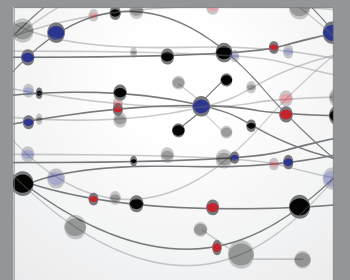

The Scientific World Journal
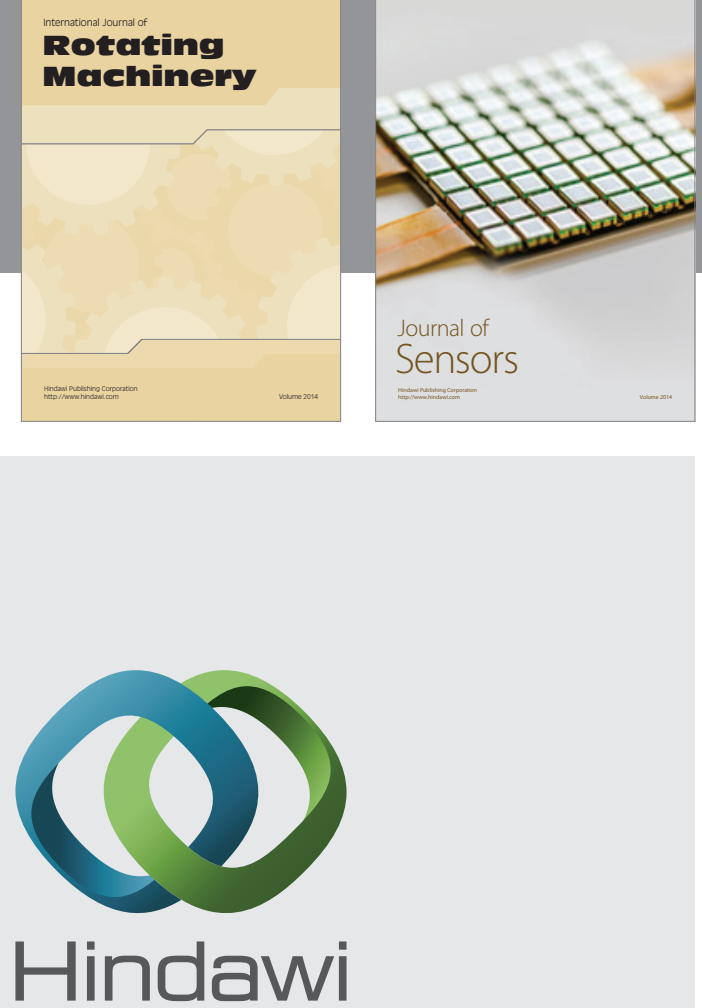

Submit your manuscripts at http://www.hindawi.com
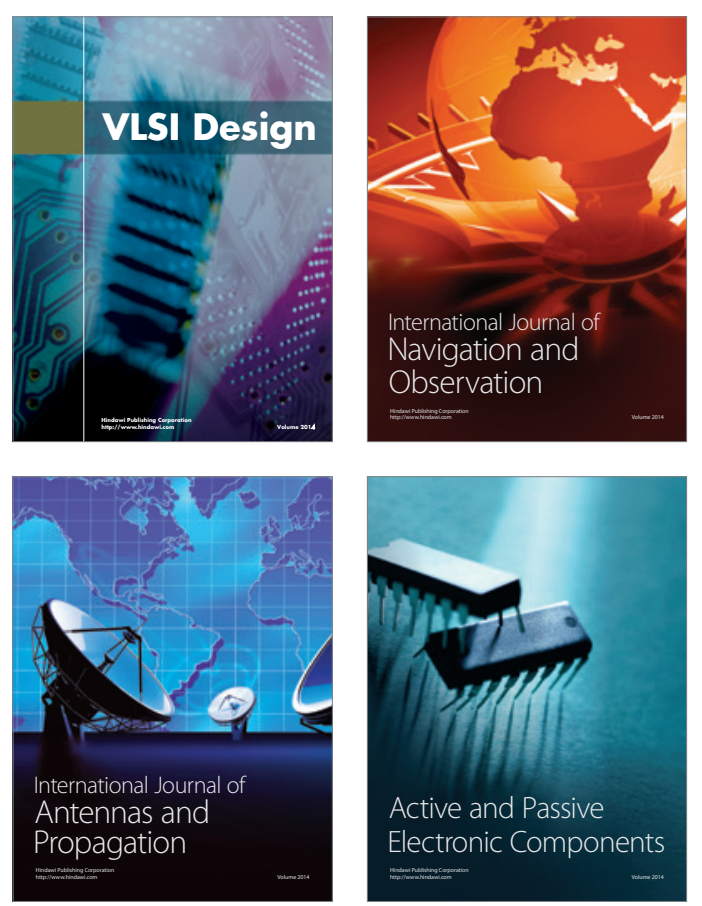
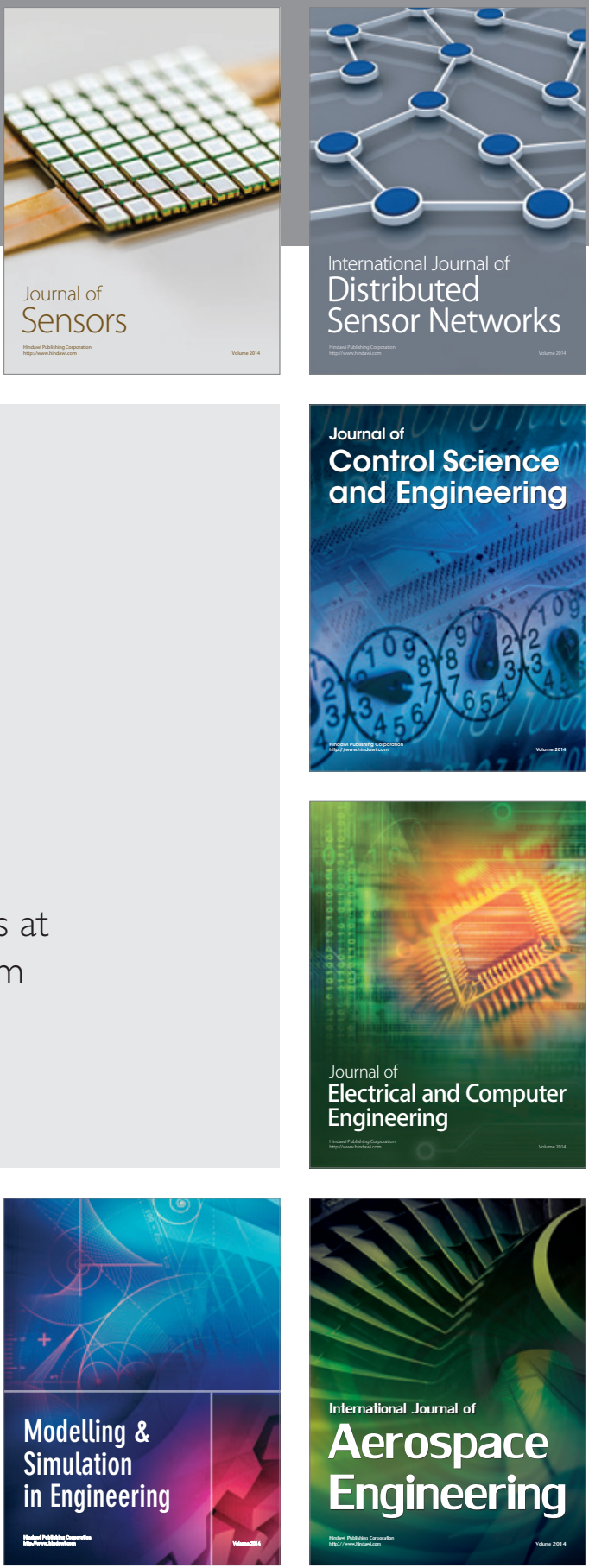

Journal of

Control Science

and Engineering
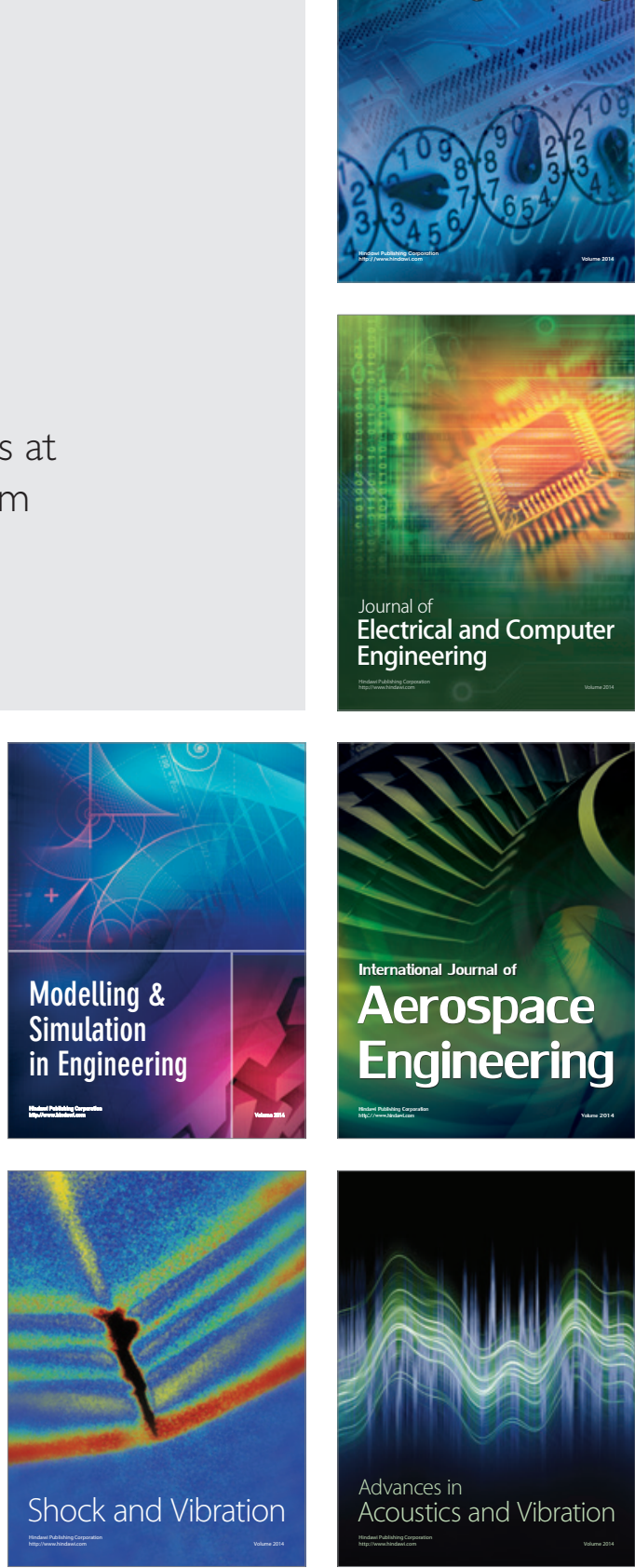